\title{
Surfaces
}

\section{CONSTRUIRE UNE POSITION D'ÉNONCIATION FÉMINISTE ET THÉOLOGIQUE. ANALYSE DE LA RÉSISTANCE DU GROUPE \\ L'AUTRE PAROLE}

\section{Denise Couture}

Volume 2, 1992

ACTES DU COLLOQUE « REPENSER LA CULTURE »

ACTS OF THE CONFERENCE "RETHINKING CULTURE"

URI : https://id.erudit.org/iderudit/1065227ar

DOI : https://doi.org/10.7202/1065227ar

Aller au sommaire du numéro

Éditeur(s)

Les Presses de l’Université de Montréal

ISSN

1188-2492 (imprimé)

1200-5320 (numérique)

Découvrir la revue

Citer ce document

Couture, D. (1992). CONSTRUIRE UNE POSITION D’ÉNONCIATION FÉMINISTE

ET THÉOLOGIQUE. ANALYSE DE LA RÉSISTANCE DU GROUPE L'AUTRE

PAROLE. Surfaces, 2. https://doi.org/10.7202/1065227ar

\section{Résumé de l'article}

La subjectivité féminine dans le discours théologique : étude des pratiques de résistance des groupes féministes au Québec. Mise en évidence de la notion de sujet en transit de de Certeau.
Copyright (C) Denise Couture, 1992

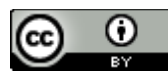

Ce document est protégé par la loi sur le droit d'auteur. L'utilisation des services d'Érudit (y compris la reproduction) est assujettie à sa politique d'utilisation que vous pouvez consulter en ligne.

https://apropos.erudit.org/fr/usagers/politique-dutilisation/ 
CONSTRUIRE UNE POSITION D'ÉNONCIATION FÉMINISTE ET THÉOLOGIQUE

\author{
ANALYSE DE LA RÉSISTANCE DU GROUPE L'AUTRE PAROLE
}

Denise Couture

Cette conférence a été donnée au colloque

Repenser la culture

qui s'est tenu à l'Université de Montréal, les 3, 4 et 5 avril 1992.

Les organisateurs tiennent à remercier, pour leur soutien financier:

le Conseil de Recherche en Sciences Humaines du Canada

la Faculté des Arts et des Sciences, Université de Montréal

le Vice-rectorat à l'enseignement et à la recherche, Université de Montréal

le Département de Littérature comparée, Université de Montréal Alitalia

\title{
RÉSUMÉ
}

La subjectivité féminine dans le discours théologique: étude des pratiques de résistance des groupes féministes au Québec. Mise en évidence de la notion de sujet en transit de de Certeau.

\section{ABSTRACT}


The problem of female subjectivity in religious discourse: a study of the resistant practices of feminist groups in Québec, with reference to de Certeau's concept of transitory subjectivity.

L'émergence des 'études culturelles' n'a pas eu pour effet une élévation de la 'culture' au rang d'un nouvel objet de savoir. Le mot 'culture' est plutôt devenu un marqueur, un signe, qui ouvre un champ de possibilités pour analyser autrement la situation dans laquelle on est plongé. Veut-on trouver une définition à ce mot? Johnson répond:

'Culture' has value as a reminder but not as a precise category; Raymond Williams has excavated its immense historical repertoire. There is no solution to this polysemy: it is rationalistic to think we can say 'henceforth this term will mean...' (1986-1987: 43).

On ne cherche pas ici la plus juste adéquation possible entre le mot et la chose. Le mot 'culture' acquiert plutôt une fonction d'orientation pour l'analyse. Une fonction de rappel, selon Johnson: le rappel, par exemple, d'un mode d'analyse qui ne confine pas, qui ne confine plus, les conditions de production et les effets du discours au statut de 'non-dit'. Le caractère 'neutre' du travail de description ne fait plus vrai. Ce qui fait vrai, en revanche, c'est que la description est déjà productrice de rapports sociaux. "Il n'est possible de dire le sens d'une situation, écrit de Certeau, qu'en fonction d'une action entreprise pour la transformer" (1974: 248-249). Dans cette ligne, Giroux propose d'analyser la culture comme "a set of activities which is lived and developped within assymetrical relations of power" (Giroux et al. 1984: 478): "a contested terrain, a site of struggle and transformation" (Giroux 1992: 202). On s'oriente ici vers un type d'analyse qui vise à décrire/modifier les enchevêtrements de rapports de force qui nous déterminent déjà. En somme, d'un parcours dans le domaine des études culturelles, je n'ai pas d'abord appris ce qu'est la 'culture'; j'y ai reconnu un ensemble de possibilités pour 'penser autrement': penser des événements, ce qui advient; penser la politique de production du savoir, les conditions d'émergence et d'exercice des discours; penser la situation dans laquelle on est plongé et qui nous fait ce que nous sommes; bref, 'penser' et 'apprendre à penser' tout à la fois.

Une telle orientation rappelle une phrase célèbre de Heidegger: "Ce qui donne le plus à penser dans notre temps qui donne à penser est que nous ne pensons pas encore" (1954: 24). Oui, peut-être, la première tâche de l'intellectuel-le est-elle encore et toujours d'apprendre à penser. Mais la formulation de Heidegger répond-elle encore aux urgences d'aujourd'hui? Ou du moins, ne pourrait-on la reprendre autrement? Après Foucault, penser, c'est résister. Je risquerais de ré-écrire ainsi la phrase de Heidegger: 
"Ce qui donne le plus à résister dans notre temps qui donne à résister est que nous ne résistons pas encore". Telle serait l'urgence du travail intellectuel: apprendre à résister et, comme professeure, faire apprendre à résister, dans un monde où, sur le fond d'une désespérance culturelle, s'est installé un désengagement ambiant devant la trop immense tâche de changer le monde. En n'oubliant pas la co-originarité du 'penser' et du 'résister', on peut dire avec Hall:

The vocation of cultural studies has been to enable people to understand what is going on, and especially to provide ways of thinking, strategies for survival, and resources for resistance to all those who are now -- in economic, political, and cultural terms -- excluded from anything that could be called access to the national culture of the national community (1990: 22).

Je propose cette fiction: 'nous', féministes et chrétiennes, 'nous' en sommes à apprendre la résistance aux multiples utilisations du pouvoir contre nos corps de femmes par d'autres, par nous-mêmes, par la société et ses institutions, par les Églises. Comment peut advenir une telle résistance féministe et théologique? Quelles sont ses possibilités, ses visées, ses dynamiques? On peut commencer à répondre à ces questions avec le Foucault qui a montré que le discours (par exemple, celui que l'on peut tenir sur la résistance féministe et théologique) et le sujet (par exemple, le sujet de cette résistance) sont, tous deux, des produits, des effets du pouvoir. "Le pouvoir est omniprésent", écrit Foucault. Comme "multiplicité de rapports de force", il "se produit à chaque instant", il "est partout", "il vient de partout". Pouvoir et savoir sont liés, entremêlés, de sorte qu'aucun savoir ne peut se développer dans un champ libre par rapport au pouvoir: de sorte que les discours "sont des éléments ou des blocs tactiques dans le champ de rapports de force" (Foucault 1976: 121-122.134). On peut prendre la parole sur tel ou tel sujet d'intérêt 'en tant que théologienne féministe', le discours n'en demeure pas moins le produit d'un ensemble de rapports de force. Il ne s'agit plus simplement de parler, donc, mais aussi d'examiner ce qui rend possible cette parole et dans l'intérêt de qui. Dans le domaine des études culturelles, écrit Rooney, "theory is understood primarily as the practice of interrogating the production of knowledge" (1990: 16). Si Foucault a voulu montrer que nous sommes plus libres que nous ne le pensons (Foucault 1988: 10), c'est qu'il est possible de jouer sur les effets du pouvoir: de produire des discours tactiques et de construire des conditions de subjectivation; bref, de résister.

Comme voie pour l'analyse d'une résistance féministe et théologique, je choisis ici de travailler à la construction d'une position d'énonciation, i.e. de porter attention aux conditions d'émergence et d'exercice de ce discours de résistance. Il s'agit de dé-construire ce dont le discours est un effet: de permettre, de rendre possible la résistance. Probyn fait un lien entre la construction d'une position d'énonciation et les technologies de soi de Foucault. Dans les deux cas, il y a construction de soi par soi (Probyn 1992: 504). On peut ajouter: dans les deux cas, il y a préparation à la capacité de faire jouer la "productivité tactique" du discours en faveur de la résistance. 
Je proposerai trois opérations de la construction d'une position d'énonciation féministe et théologique.

Une précision d'abord à propos de la position d'énonciation. Il ne faut pas la confondre avec un 'lieu propre' d'où l'on parle. Par exemple, la place que sa spécialité a valu à l'expert universitaire. Comme le calcul tactique, la position d'énonciation "ne peut compter sur un propre, ni sur une frontière qui distingue l'autre comme une totalité visible" (de Certeau 1980: 21; voir aussi Probyn 1992: 506). Il est possible de se construire, de construire ce à partir de quoi on pourra parler, mais seulement là où le 'je' ne peux pas pointer du doigt un 'propre' et dire: voici c'est exactement ici que se trouve ma place et elle est distincte de celle des autres 'je' en présence.

La position d'énonciation vise, ici, une tournure d'être, un pli, un mode de rapports entre soi et les mots et les situations. Dans la mesure où cette tournure d'être ne qualifie pas un 'je' isolé sur lui-même ou sur elle-même qui entre après coup en relations avec les autres, on pourrait l'appeler aussi une 'tournure d'événements' à laquelle le 'je' participe. Une telle position d'énonciation se forme, se forge, se construit. Elle émerge d'un réseau de relations, non pas compris comme 'lieu propre', mais plutôt comme 'temps' d'existence qui précède le partage entre soi et l'autre.

Faut-il revenir au risque de la communauté, de l'appartenance à un réseau de relations en construction? Du moins, on peut prendre le risque, celui de petites communautés qui fabriquent collectivement de la vérité en la faisant circuler sans point d'arrêt et sans point d'origine, là où "la relation est le lieu existentiel" (de Certeau/ Domenach 1974: 34).

Première opération de la construction d'une position d'énonciation, donc: un 'se laisser forger une tournure d'être' par l'appartenance à une communauté. Le 'ce à partir de quoi' on pourra parler est un 'nous'. Je fais fonctionner cette opération en pensant ce 'nous' comme un groupe de résistance local, L'autre Parole, une ecclesia de femmes québécoises, féministes et chrétiennes. Ce 'nous', le réseau de relations de l'ecclesia, forme à une tournure d'être et de penser que nous construisons ensemble, à laquelle nous nous forgeons ensemble et qui construit une manière singulière de répondre et de parler des événements qui nous arrivent. Ce qui est appris de l'ecclesia, par chacune, ce n'est ni un système cohérent d'idées, ni un point de vue sur le réel; mais 'ce à partir de quoi on pourra parler' comme féministe et chrétienne. La théologie féministe n'est plus une approche méthodologique, féministe, appliquée au domaine d'objets délimité par la discipline de la théologie, mais un pli de l'être qui marque les rapports aux événements: une formation du corps, dans le corps, à une attention portée aux traumatismes vécus par des femmes parce qu'elles sont des femmes /pp. 8-9/ (position d'énonciation féministe) et à une attention portée à l'expérience de Dieue, aux conditions d'exercice et aux effets de la circulation du mot 'Dieu' (position d'énonciation théologique; elle est chrétienne parce que nous sommes déjà formées à la manière chrétienne de parler de Dieue). Une position d'énonciation, en somme, comme un 'se laisser forger' par la tournure d'un 'nous'. 
Un danger de cette opération: que le 'parler à partir de l'ecclesia' ne cache un 'parler pour les autres' aux couleurs hégémoniques. L'une des tactiques de L'autre Parole consiste à fabriquer de la vérité autrement, à ne pas parler "dans le vrai" pour le dire à la manière du Foucault de L'ordre du discours, si bien que par l'ininterrompue circulation de la vérité, la construction par l'une de toutes les autres est chaque fois en jeu. La position d'énonciation vise "a 'common ground of enunciation'", selon l'expression de Probyn. Celle-ci ajoute: "this is not to say that one represents all; rather, that in technologizing one's self, one necessarily works on and for the selves of others as well" (Probyn 1992: 509). En tant qu'événement que l'une ou l'autre, les unes ou les autres, ne peuvent maîtriser comme 'je' isolée des autres, le 'nous' prend la figure d'un 'commun en transit'. Le risque du transit de l'ecclesia correspond et répond au risque, pour chacune, de la construction de soi par l'ecclesia. C'est ce risque que je peux prendre, comme théologienne féministe, celui de laisser l'ecclesia de femmes construire un soi que je peux faire jouer dans le travail universitaire. Le féminisme et la théologie ne sont plus, respectivement, une approche méthodologique et un champ disciplinaire, mais plutôt des positions d'énonciations, des modes de rapport aux événements ou, plutôt, des 'tournures d'événements' qui construisent le soi. Un soi qui, au ras de la quotidienneté, porte attention à telle chose plutôt que telle autre, qui participe au langage de telle manière et qui agit de telle manière. Ces tournures singulières émergent du 'commun en transit' et annoncent la résistance à une politique du savoir qui individualise le sujet connaissant, le rend propriétaire de ses connaissances et donne, dans l'échange, les résultats de la théologie féministe toujours perdants.

Il s'agit de nous former à une manière de fabriquer de la vérité autrement. Deleuze a appelé l'un des quatre plissements de la construction de soi par soi chez Foucault "le pli du savoir" ou "pli de vérité" (Deleuze 1986: 111, 122). Il "constitue, écrit-il, le rapport du vrai à notre être, et de notre être à la vérité, qui servira de condition formelle à tout savoir, à toute connaissance" (Deleuze 1986: 111). Ce rapport, comme pli, singularise la position d'énonciation du groupe de résistance local. Comment émerge, plus précisément, le 'pli de vérité' appris de la participation à l'ecclesia L'autre Parole?

D'une analyse de la manière de faire circuler la vérité entre 'nous' (voir L'autre Parole 1991: 23ss.), il ressort que la vérité se décide au lieu de l'émergence de la parole. Il ne s'agit pas de distinguer le vrai de son contraire, le faux. La question de la vérité se joue plutôt dans la distinction entre ce qui est digne d'être dit et ce qui n'est pas digne d'être dit et donc n'est pas dit. J'illustrerai ceci à partir d'un récit de la poétesse France Daigle. Elle raconte l'histoire d'une femme qui commence à trouver des mots pour dire une blessure demeurée longtemps occultée. La prise de 
parole est tissée d'avancées et de reculs, de dévoilements et d'oblitérations. Elle le sait. Elle sait que le 'parler' est un chemin d'exister. La mémoire et l'oubli scandent le récit de Daigle. La femme trouve des mots pour dire la blessure; puis, elle oublie, elle oublie l'oubli; puis, il arrive que les mots, les mots troublants, émergent à nouveau; puis, elle oublie encore. Ainsi va l'histoire. Elle oublie quand elle ne sait plus si c'est elle qui dit ce qu'elle dit, quand elle ne voit plus comment ce qu'elle dit peut produire son existence. Le 'parler' exige un travail sur soi:

Elle parle mais quelque chose en elle se brise à mesure que les mots sortent de sa bouche. Elle se resserre, se ramène plus près d'où elle pourra encore parler. Elle marche, vit ainsi pendant quelques heures, quelques jours. Quelques nuits même. Puis, petit à petit, cela prend une forme de permanence, cela devient elle (Daigle 1991: 14).

Elle parle d'une blessure. "Quelque chose en elle se brise". Celle qui parle doit se déconstruire et se construire autrement pour être capable de continuer à marcher sur le chemin de cette prise de parole. Alors, elle vise une tournure d'être "plus près" du lieu d'émergence de sa parole. "Elle pourra peut-être encore parler". Sa tâche: désapprendre l'habituel, l'accoutumé, l'évident; apprendre la tournure d'être que produit la parole. "Elle marche, vit ainsi". Elle s'habitue à être, à savoir par elle-même ce qu'elle dit... et si cela est possible, "petit à petit, cela prend une forme de permanence". Elle change. "Cela devient elle".

On peut reconnaître dans cette deuxième opération de la construction d'une position d'énonciation, i.e. de 'ce à partir de quoi on pourra parler', ce que Probyn appelle une articulation alternative "of the non-discursive to the discursive which then plays back again into the non-discursive" (505). Cette 'manière de parler' produit un 'soi en transit'. Elle fait désapprendre l'ethos du travail intellectuel en sciences humaines qui exige la maîtrise du savoir. Le sujet, construit par cet ethos scientifique, doit pouvoir utiliser son savoir sans hésiter, sans douter, sans avoir à reconsidérer, chaque fois, les procédés de production. Cela, le 'soi en transit' n'a pas les moyens de le faire. C'est au-delà de son pouvoir. "One of the first thing to do is to think within the limits of one's power", écrit Spivak (1989: 147). Le 'soi en transit' ne peut abstraire son savoir du chemin d'exister: le transformer en 'opinions' qu'on pourrait articuler en système organisé de pensée et auquel on pourrait référer au besoin; le tourner en 'somme de connaissances' disponible pour un découpage et une transmission en petites tranches. Mais le 'soi en transit' peut parler autrement et ainsi produire de nouveaux rapports de force.

Comment ça se passe? Dans un 'raconter/soupçonner'. Les mots partagés entre nous appellent l'écoute... L'écoute de quelque chose qu'on nous a habituées à ne pas voir, à ne pas dire, à ne pas entendre; l'écoute des blessures racontées par des femmes; l'écoute de ses propres blessures comme d'un écart en soi d'avec soi. Nous racontons, écoutons des histoires, celles qui blessent, celles qui sont jugées insupportables, celles qui ne 
s'expliquent pas. Nous les tenons pour vraies, car ces histoires qu'on nous a habituées à tenir pour banales, anodines et sans intérêt, sont ce qui est digne d'être dit. L'ecclesia ouvre un espace collectif pour dire/ écouter ces histoires. Le 'raconter' est un moment d'émergence de la parole, d'étonnement, de douleur et de rage partagés. Tout cela et nos rires, nos pleurs, nos colères, nos frissons dans le dos, nos serrements de la gorge prennent part à notre rapport à la vérité.

Dans le 'raconter', résonne un 'soupçonner'. Les mots partagés suscitent une quête incessante... La recherche de ce qui rend possible les blessures racontées. Nous faisons jouer l'hypothèse que l'androcentrisme détermine nos vies dans TOUT ce qui va de soi: dans les mouvements des yeux et du corps, dans les manières coutumières d'être ensemble, dans le partage des tâches au quotidien, etc. Nous savons, écrit Guillaumin, qu'à chaque fois, "ce que nous avons sous les yeux, nous ne le voyons pas" (1978: 21).

Le 'soupçonner' détourne l'attention des fautes morales, des manques de capacité, de valeur, de mérite et fait oeuvre de déculpabilisation pour les violences subies. Les blessures racontées apparaissent comme des effets des pratiques sociales. Il n'y a plus un sujet autonome, un sujet de vouloir et de pouvoir, coupable ou dans l'erreur, mais un soi à déprendre de ce qui fait qu'elle est ce qu'elle est. Le 'soupçonner' donne la direction du 'parler ensemble'. Plus que deux moments consécutifs, le 'raconter' et le 'soupçonner' se chevauchent partout comme s'y enchevêtre déjà le 'changer' du 'soi en transit'. Le pli du savoir importe plus ici que les choses sues. Il rend possible l'émergence d'une nouvelle parole, comme d'une nouvelle traversée à la surface des évidences désormais vacillantes.

Il y a, par exemple, la tactique d'écrire le mot Dieue avec un 'e' à la fin. Je m'y arrêterai car l'analyse de cette pratique discursive permettra d'illustrer le pli du savoir du 'raconter-soupçonner-changer' et de montrer, aussi, comment s'y joue la primauté du mode du savoir sur les choses sues. Dieue avec un 'e', cela commence en septembre 1988 au colloque annuel de L'autre Parole (voir L'autre Parole 1988). Le thème du colloque, "Les images de Dieu au féminin", invitait à imaginer une voie possible pour dire Dieue à partir de nos corps de femmes. Si, comme l'a écrit Christine Delphy, le féminisme procède "à partir de perceptions /pp. 12-13/ pré-existantes de ce qui est supportable et de ce qui ne l'est pas, de ce qui est juste et de ce qui ne l'est pas" (Delphy 1981: 70), on peut dire que nous sommes parties du caractère intolérable des images masculines du Dieu chrétien, surtout du Dieu Père, Patriarche. Comme on le sait, l'Église catholique est le dernier bastion institutionnel, en Occident, qui ne reconnaît pas encore des droits égaux aux hommes et aux femmes à l'intérieur de sa propre organisation (Roy 1990; Dumais 1992). Mais les femmes chrétiennes doivent de plus, du fond de leur expérience spirituelle et tout au long de leur vie, prier un Dieu de l'autre genre auquel on a donné, de surcroît, l'image du Grand Patriarche. C'est trop! C'est un obstacle de fond à la possibilité de parler de Dieu à partir de nos histoires, de nos blessures, de nos corps de femmes. Dire Dieu au masculin, dire Dieu, d-i-e-u, cela n'est plus possible. 
Au colloque de 1988 donc, une trentaine de femmes sont rassemblées. Elles ouvrent la possibilité, pour elles, de pouvoir continuer de parler de Dieue. Elles cherchent une manière de faire qui permettrait à chacune de pouvoir parler à partir de son histoire de femme. Les mots, la vérité, circulent... L'intolérable est raconté. On ne s'entend pas sur une voie possible. On continue de parler. Puis, une ouverture se crée: Dieue avec un 'e' émerge comme une possibilité d'où chacune pourra continuer de parler de Dieue, raconter les blessures, déconstruire ce qui les rend possibles et ouvrir de nouvelles plages pour la créativité.

Ce choix de l'ecclesia, dont je suis solidaire, m'a étonnée depuis le début. Pour quoi ce 'e' planté après les d-i-e-u du mot 'Dieu'? Que vise cette pratique discursive? Plusieurs autres possibilités étaient ouvertes. On aurait pu remplacer le mot blessant, Dieu, par un silence tenace; ou conserver seulement l'adjectif 'divin' pour qualifier certains événements auxquels nous prenons part (Welch 1985); remplacer le mot par son féminin grammatical, 'Déesse' (Daly 1978; Christ 1987); faire circuler le mot composé 'DieuDéesse' (Ruether 1981); ou encore profiter d'une possibilité que donne la langue française et écrire 'Dieu-e' (L'autre Parole, parfois, avant 1988).

Bien sûr, dans tout cela, il n'y a pas de concours de bonne réponse. Il ne s'agit pas de décider qui a réussi à représenter le plus adéquatement possible la 'réalité' de Dieue. Dieue, Dieu-e, Dieu-Déesse, Déesse, le divin ou le silence, chacun de ces énoncés émerge d'une pratique féministe particulière, d'un mode singulier de résistance à la tradition chrétienne et théologique: on ne peut pas les comprendre, ces énoncés, en soi, faisant abstraction de la pratique discursive qui les permet. $\mathrm{D}$ 'où cette règle de précaution pour l'analyse: ne pas partir seulement de la chose dite, Dieue, mais aussi des conditions d'émergence et d'exercice de l'énoncé. Autrement dit, il ne suffit pas de dégager un ensemble d'idées féministes et théologiques, propres au groupe L'autre Parole et, de là, induire ou déduire les raisons qui ont conduit au choix d'écrire Dieue avec un 'e'. Il faut aussi analyser un mode singulier de production de vérité.

La tactique d'écrire le mot Dieue avec un 'e' émerge de la pratique discursive du 'raconter-soupçonner-changer'. Dieue avec un 'e', cela étonne, cela surprend. La féminisation graphique a une fonction de rappel au soupçon de toutes les utilisations de Dieu. Elle remplira sa fonction aussi longtemps que le petit 'e' étonnera; aussi longtemps qu'il rappellera la tâche constante de désapprendre les tournures d'être trop bien apprises. Le mot ne réfère pas à la 'réalité' de la 'chose' Dieue. Ce qu'est Dieue? Là n'est pas la question. Il ne s'agit pas de décider s'il, elle, est...; s'il, elle, est masculin ou féminin. Dieue, un mot-effet, une construction grammaticale dont la fonction est d'avoir un effet sur soi, sur la construction de soi. Écrire Dieue avec un 'e', cela ne dit encore à peu près rien de Dieue. Sinon, par la négative, que Dieue n'est pas un Père, qu'elle n'est pas de genre masculin. 
Comme on le sait, toutes nos images de Dieu sont des projections humaines. L'opération de déconstruction des images masculines de Dieu contraint, semble-t-il, à choisir parmi les projections humaines, soit le masculin (Dieu), soit le féminin (Déesse, Dieue), soit un équilibre des deux (Dieu-Déesse, Dieu-e). Pour éviter que la thématique du féminin-masculin ne gère les options de féminisation du mot Dieu et n'occulte le mode de production du savoir, on peut, avec Maurice Boutin, distinguer deux modes de projection: l'une objectivée, l'autre non objectivée. Dieu, selon Boutin, est une projection non objectivée: une projection de ce qui, du fond de l'expérience humaine, demeure au-delà de toute objectivation possible. Je comprends l'énoncé 'Dieue' comme la projection d'une position d'énonciation, bien sûr, non objectivable. Le féminin de Dieue ne réfère pas au genre; il a plutôt une fonction de rappel de l'événement du 'raconter-soupçonner-changer'. Le mot 'Dieue' peut être compris comme la projection non objectivée d'un mode d'être en commun et, dans ce sens, il a pour effet de tourner l'ecclesia vers la construction de la position d'énonciation: d'un 'commun en transit' et d'un 'soi en transit'. Dieue, le commerce de ce mot, a la force de tirer l'ecclesia vers le 'ce à partir de quoi' et le 'ce vers quoi' de sa résistance, vers l'autre, vers l'absent-e, vers ce qui lui manque et la fait marcher. L'énoncé prend son sens dans son émergence et par ses effets. Je pense qu'on ne peut le comprendre, dans sa singularité, si on le coupe du pli de la vérité qui le permet.

J'aborderai la troisième opération de la construction de la position d'énonciation en notant ceci: le nom de l'ecclesia, L'autre Parole, lui donne la place de l'autre, de celle qu'il faut écouter; de celle qui a quelque chose à apporter aux autres; de celle, peut-être, qui est 'plus proche de la vérité' parce qu'elle analyse la situation à partir du 'point de vue' privilégié des 'opprimées'. D'où le danger de l'oubli de son autre: le danger de la réification du groupe ou de l'appartenance au groupe. C'est une question délicate en ce temps où plusieurs théoriciennes féministes défendent, contre ce qu'on appelle le 'féminisme postmoderne', la nécessité d'une lutte politique concertée (voir Felski 1989; Harstock 1989; Macia-Lees et all. 1989). Comment penser ce genre de lutte à partir d'un groupe de résistance qui ne se donnerait plus la place de l'autre à écouter, mais qui serait plutôt dans l'attente d'apprendre de son autre, en soi, en l'autre? Car, dans la fiction que je fais jouer ici, c'est cette possibilité qui annonce la plus grande force transformatrice des rapports sociaux.

Il faut revenir à la question: 'comment se produire comme sujet?' Non plus seulement 'comme sujet du savoir', mais, cette fois, 'comme sujet de la transformation sociale'. Deleuze pose la question ainsi: "A quel pouvoir prétendre et quelles résistances opposer?" (Deleuze 1986: 122). Réponse: prétendre au pouvoir et opposer la résistance de la construction d'un 'commun en transit' et d'un 'soi en transit'. Tâche qui nécessite à nouveau un travail sur soi; le soi ainsi construit n'est ni le sujet autonome du savoir maîtrisé, ni celui de l'action planifiée. Cela exige la démaîtrise de l'idée courante de la responsabilité selon laquelle le sujet est responsable de ses actions s'il peut garantir l'efficacité de ses projets préalables. Selon Welch, 
une auteure qui propose une théologie féministe de la résistance, la fiction de l'action planifiée -- et, autant que possible, réussie -- est, par définition, légitimatrice du statu quo. Car, à la fin, n'est efficace qu'un plan d'action qui s'inscrit dans le jeu préétabli par les règles du système. Les actions en vue de changements structurels réussissent peu ou ne réussissent pas. Elles sont presque irresponsables (Welch 1990: 104). La construction d'un 'commun en transit' et d'un 'soi en transit' demande la non-maîtrise de ce que sera demain parce qu'elle est en même temps non-maîtrise des autres, mais surtout parce qu'elle ploie les rapports de force en soi comme un 'pouvoir construire' un soi en train de se déprendre d'une politique du savoir qui continue de favoriser l'occultation de l'intolérable raconté et d'une politique de l'action qui fait sauter par-dessus des possibles pour soi, maintenant.

Le transit de la résistance laisse à d'autres, aux autres qui viennent avec nous et après nous, le soin de préparer l'avenir. À L'autre Parole, cet à-venir se joue, il se construit maintenant dans un 'faire église' à partir de nos corps de femmes. Nous pouvons construire une église de femmes qui ne se situe, ni 'dans' l'Église, ni 'hors' de l'Église catholique. Une église qui permet le risque de la construction de soi, autrement. Des 'prêtresses', chacune, l'une ou l'autre, qu'on n'appelle pas des prêtresses, créent et animent les célébrations symboliques. Nancy Fraser a raison. Avec la résistance, on ne peut que 'préparer' un temps nouveau (1989: 59); mais le 'préparer', c'est le faire maintenant. C'est ne pas attendre pour concrétiser un possible pour soi, pour 'nous'. À ce pouvoir, nous pouvons prétendre. La position d'énonciation, écrit Probyn, "expresses the potential for articulating 'wishes' to 'facts', of turning wishes into des faits accomplis" (1992: 508).

Je suis partie avec Foucault de l'idée que nous sommes plus libres que nous ne le pensons. En effet, nous pouvons déconstruire le 'je' isolé, le 'je' du savoir maîtrisé ou de l'action planifiée; nous pouvons fabriquer de la vérité autrement et produire de nouveaux rapports de force. J'ai voulu éprouver la possibilité, pour l'intellectuelle, de travailler, comme à rebours, sur ce qui produit son discours en vue de se déprendre d'une politique du savoir qui défavorise ou qui bloque sa propre résistance. D'une telle démarche, nulle assurance ne peut résulter, seulement le défi d'apprendre la résistance en la risquant, en la faisant.

À partir d'une résistance locale (peut-on analyser la résistance autrement?), j'aurai retenu trois opérations de la construction d'une position d'énonciation féministe et théologique: se recevoir d'un 'commun en transit'; construire un 'soi en transit'; et vivre à la mesure d'un pouvoir être maintenant. C'est trois fois un mode de rapport à l'autre (en soi, en l'autre) qui est en jeu. C'est aussi la préparation d'une 'théologie féministe' telle que je l'imagine à l'université. Projet impossible sans les autres... "Ce que je fais de plus vrai, je ne le puis sans toi -- mais je ne peux pas te ramener à ce que je fais ou à ce que je sais" (de Certeau 1987: 113). 


\section{Denise Couture}

Faculté de théologie

Université de Montréal

Surface Page d'Acceuil/Home Page

\section{Références}

BOUTIN, Maurice (1988): "Dieu et la projection non-objectivée.

Conséquences de la compréhension de Dieu dans la théologie de Rudolf Bultmann." Laval Théologique et Philosophique 44/2 (juin 1988): 221-246.

CHRIST, Carol P. (1987): Laughter of Aphrodite, Reflections on a Journey of the Goddess San Francisco: Harper and Row, 1987, 240 p.

DAIGLE, France (1991): La beauté de l'affaire, Fiction autobiographique à plusieurs voix sur son rapport tortueux au langage. Outrement: Éd. nbj, 54 p.

DALY, Mary (1978): Gyn/ecology, The Metaethics of Radical Feminism. Boston: Beacon Press, 1978, 485 p.

DE CERTEAU, Michel et Jean-Marie DOMENACH (1974): Le christianisme éclaté. Paris: Seuil, $118 \mathrm{p}$.

DE CERTEAU, Michel (1974): La culture au pluriel, Paris: Union générale d'éditions, $313 \mathrm{p}$.

- -- (1980): L'invention du quotidien, T. 1: Arts de faire, Paris: Union générale d'éditions, $375 \mathrm{p}$.

--- (1987): La faiblesse de croire, Paris: Seuil, 322 p.

DELEUZE, Gilles (1986): Foucault, Paris: Les éditions de Minuit, 141 p.

DELPHY, Christine (1981): "Le patriarcat, le féminisme et leurs intellectuelles": Nouvelles Questions Féministes (1981 -- no. 2) 59-74; p. 70.

DUMAIS Monique (1992): Les droits des femmes, [Collection Interpellations, 2], Montréal: Éd. Paulines, 132 p.

FELSKI, Rita (1989): "Feminism, Postmodernism and the Critique of Modernity": Cultural Critique 13 (1989) 33-56. 
FOUCAULT, Michel (1971): L'ordre du discours, Paris: Gallimard, $81 \mathrm{p}$.

--- (1976): La volonté de savoir, Histoire de la sexualité, T. 1, Paris: Gallimard, $211 \mathrm{p}$.

- -- (1988): Rux Martin, "Truth, Power, Self: An Interview with Michel Foucault", dans: L. H. Martin, H. Gutman et P.H. Hutton (dir.), Technologies of the Self, A Seminar with Michel Foucault, London: The University of Massachusetts Press, 166 p.; pp. 9-15.

FRASER, Nancy (1989): Unruly Practices, Power, Discourse, and Gender in Contemporary Social Theory, Minneapolis: University of Minnesota Press, $201 \mathrm{p}$.

GIROUX, Henry, David SHUMWAY, Paul SMITH et James SOSNOSKI (1984): "The Need for Cultural Studies: Resisting Intellectuals and Oppositional Public Spheres": Dalhousie Review 64 (1984) 472-486.

GIROUX, Henry (1992): "Resisting Difference: Cultural Studies and the Discourse of Critical Pedagogy", dans: L. Grossberg, C. Nelson et P.A. Treichler (dir.), Cultural Studies, New York, Routledge, 788 p.; pp. 199-212.

GUILLAUMIN, Colette (1978): "Pratique du pouvoir et idée de Nature. 1) L'appropriation des femmes": Questions Féministes 1/2 (1978) 1-30.

HALL, Stuart (1990): "The Émergence of Cultural Studies and the Crisis of the Humanities": October 53 (1990) 11-23.

HARTSOCK, Nancy (1989): "Postmodernism and Political Change: Issues for Feminist Theory": Cultural Critique 14 (1989) 15-33.

HEIDEGGER, Martin (1954): Qu'appelle-t-on penser? Paris: Presses Universitaires de France, 1973 (1954), 262 p.

JOHNSON, Richard (1986-1987): "What is Cultural Studies Anyway?": Social Text 16 (1986-1987) 38-80.

L'AUTRE PAROLE (1988): Dieue au féminin (déc. 1988 -- no. 40) 35.

- --, (1991): ThéAlogie, (sept. 1991 -- no. 51) 39.

MARCIA-LEES, Frances E., Patricia SHARPE et Colleen BALLERINO COHEN (1989): "The Postmodernist Turn in Anthropology: Cautions from a Feminist Perspective": Signs 15/1 (1989) 7-33.

PROBYN, Elspeth (1992): "Technologizing the Self: A Futur Anterior for Cultural Studies", dans: L. Grossberg, C. Nelson et P.A. Treichler (dir.), Cultural Studies New York: Routledge, 788 p.; pp. 501-511.

ROY, Marie-Andrée (1990): "Le changement de la situation des femmes dans le catholicisme québécois": Sociologie et Sociétés 22/2 (oct. 1990) 95-114. 
ROONEY, Ellen (1990): "Discipline and Vanish: Feminism, Resistance to Theory, and the Politics of Cultural Studies": Differences, 2/3 (1990) 14-28.

RUETHER, Rosemary Radford (1981): "La féminité de Dieu. Un problème dans la vie religieuse contemporaine": Concilium (1981 - -- no. 163) 93-101.

SPIVAK, Gayatri Chakravorty (1989): "Interview: Gayatri Chakravorty Spivak with Ellen Rooney": Differences 1/2 (1989) 124-156.

WELCH, Sharon D. (1985): Communities of Resistance and Solidarity, A Feminist Theology of Liberation, New York: Orbis Book, 102 p.

- -- (1990): A Feminist Ethic of Risk, Minneapolis: Fortress Press, 206 p. 